\title{
Impact of the Normalized Copy Number of $B C R-A B L$ Transcript upon Diagnosis on Prognosis in CML Patients Treated with Imatinib-Mesylate
}

\author{
Christophe Martinaud $^{1}$, Aurélie Mayet ${ }^{2}$, Sophie Bousquet ${ }^{3}$, Nathalie Beaufils ${ }^{4}$, Jean Gabert ${ }^{4}$, \\ Sophie Raynaud ${ }^{3}$, Marie-Joelle Mozziconacci ${ }^{5}$
}

${ }^{1}$ Fédération des Laboratoires, HIA Percy, Clamart, France; ${ }^{2}$ Département d'Epidémiologie et de Santé Publique, Ecole du Val-de-
Grâce, Paris, France; ${ }^{3}$ Laboratoire d’Onco-hématologie, Hôpital Pasteur, CHU de Nice, Nice, France; ${ }^{4}$ Laboratoire de Biologie Mo-
léculaire, APHM Nord, Marseille, France; ${ }^{5}$ Laboratoire de Biologie Moléculaire, Département de Biopathologie, Institut Paoli Cal-
mettes, Marseille, France.
Email: christophe.martinaud@inserm.fr

Received September $26^{\text {th }}$, 2011; revised October $30^{\text {th }}$, 2011; accepted November $16^{\text {th }}, 2011$.

\begin{abstract}
Quantification of the BCR-ABL transcript is recommended to follow-up CML patients treated by imatinib mesylate (IM). Results are expressed as a normalized copy number (NCN) of BCR-ABL. We studied a cohort of $98 C M L$ patients under IM as a first treatment and monitored by RQ-PCR after 12, 18 and 24 months according to the European LeukemiaNet recommendations. Our results support the hypothesis of an independent correlation between BCR-ABL NCN at diagnosis and major molecular response at 18 and 24 months in an inverse relationship. We also highlighted the possibility to use the NCN at diagnosis as a warning at diagnosis, and may be useful to identify patients who could benefit of a more rigorous follow-up.
\end{abstract}

Keywords: Chronic Myeloid Leukemia, RQ-PCR, Normalized Copy Number, BCR-ABL

\section{Introduction}

Chronic myeloid leukemia (CML) is a clonal disease of the hematopoietic stem cell characterized by a reciprocal translocation $\mathrm{t}(9 ; 22)(\mathrm{q} 34 ; \mathrm{q} 11)$ that fuses the $\mathrm{ABL}$ and BCR genes located on 9q34 and 22q11 respectively. Imatinib mesylate (IM), a tyrosine kinase inhibitor, blocks the kinase activity of BCR-ABL and induces complete cytogenetic response in a majority of the patients [1,2]. Quantification of the BCR-ABL transcript by quantitative reverse transcriptase polymerase chain reaction (RQ-PCR) is recommended for accurate follow-up of treated CML patients. Results are expressed as a ratio of BCR-ABL on a control gene (normalized copy number: NCN). Based on the IRIS (International Randomized study of Interferon and STI571) trial, the concept of measuring a $\log 10$ reduction in BCR-ABL transcript from a standardized baseline value has been proposed [3]. The standardized baseline value, defined as the median of NCN for 30 untreated patients from the same laboratory, is taken to represent the BCR-ABL transcript level of $100 \%$ tumor cell burden, and the major molecular response (MMR) is defined as a 3-log or more reduction of the baseline value $(0.1 \%)$. The IRIS study demonstrated that patients who achieved a MMR in the first year had no disease progression at 5 years [1]. Lack of MMR at 18 months is qualified as suboptimal response and might lead to a dose escalation of IM or to the shift to a second generation tyrosine kinase inhibitor [4,5]. It should be very important to evaluate the prognostic and the probability of achievement of MMR since the diagnosis to allow a better and an earlier adaptation of the treatment.

\section{Patients and Methods}

In order to evaluate the prognostic impact of BCR-ABL NCN at diagnosis on the achievement of MMR as defined by the IRIS study, we compared the NCN of MBCR-ABL transcript in newly diagnosed CML patients before treatment as detected in 3 laboratories in South of France (Marseille APHM, Marseille IPC and Nice) and analysed the data of a cohort of $98 \mathrm{CML}$ patients treated 
by IM $400 \mathrm{mg} /$ day as a first line treatment, and monitored by RQ-PCR for BCR-ABL transcript after 12, 18 and 24 months of treatment in the 3 laboratories. RQPCRs were performed on peripheral blood cells according to the European Leukemia Net recommendations [6] on 3 machines: MX3000 (Stratagen), TaqMan 7900 and TaqMan 7500 (Applied Biosystems) respectively. According to Hughes et al. [6], the control gene was ABL and the standardized baseline values were 92, 125 and 110 copies of MBCR-ABL/102 copies of ABL in the 3 laboratories respectively. Results were referenced to sets of plasmids for ABL and MBCR-ABL (Ipsogen, Marseille-France). Median ABL cycle thresholds (Ct) was 25.03 (standard deviation: 1.85) and the median ABL copy number was up to 20,000 . $64 \%$ of patients were male, median age at diagnosis was 58 years (range 1-85). Sokal risk score was available for 65 patients: $25 \%$ low risk $(\mathrm{n}=16), 48 \%$ intermediate risk $(\mathrm{n}=31)$, and $28 \%$ high risk $(\mathrm{n}=18)$. Data were analysed using SAS 9.1 Software.

\section{Results and Discussion}

At diagnosis, patients were separated in two groups, depending on whether the NCN of BCR-ABL transcript at diagnosis was above (group $\mathrm{A}, \mathrm{n}=52$ patients) or under (group $B, n=46$ ) the standardized baseline value of each laboratory. At 12 months, 80 patients were eligible for analysis: 21 patients from group A (47\%) and 22 from group B (63\%) achieved a MMR. No significant relation between NCN at diagnosis and MMR at 12 months was found (Chi-square test).

At 18 months, 98 patients were eligible for analysis: 25 patients from group A (48\%) and 35 from group B (76\%) achieved a MMR. On univariate analysis, a NCN at diagnosis above the standardized baseline value was significantly associated with a 4 fold higher risk (odds ratio $(\mathrm{OR})=0.2[0.1-0.7]$ ) of non MMR at 18 months (p $=0.001$ ). The mean NCN at diagnosis was significantly lower in patients who achieved a MMR at 18 month compared with non-responders (13 vs 21 copies of MBCR-ABL/102 copies of ABL, $p=0.02$ ANOVA). Moreover, distribution of Sokal risk showed no difference between patients who had a NCN at diagnosis above the standardized baseline and those who had a NCN at diagnosis under this baseline, attesting to an homogenous repartition of Sokal score between the two groups. We also showed that there were no difference for the mean of NCN at diagnosis between the three groups of Sokal (low, intermediary and high) i.e. 22, 18 and 15 copies of MBCR-ABL/102 copies of ABL ( $p=0.6$ variance analysis). Altogether, our analysis account for an independence of NCN at diagnosis and Sokal score in the prediction of the obtention of a MMR at 18 months.

At 24 months, 86 patients were eligible for analysis: 54 (63\%) achieved a MMR: 26 from group 1 (55\%) and 28 from group B (72\%). A significant relation between NCN at diagnosis and MMR at 24 months was still assessed (chi-square test, $\mathrm{p}=0.05$ ).

Finally, as a study demonstrated the impact of the 12-month cytogenetic response on the overall survival and the probability to achieve a complete cytogenetic response (CCyR) [7], we compared the NCN values at diagnosis with the cytogenetic responses at 12 months. These data were available for 34 patients. We compared the distribution of CCyR in each subgroup: $33 \%$ of patients with NCN at diagnosis below the standardized baseline failed to obtain a CCyR, versus $38 \%$ in the NCN above it. No significant difference of distribution was observed between the groups in terms of CCyR at 12 months. We are aware that an analysis taking CCyR at 3 months could be relevant [5], though this data was not available at the time of the study.

\section{Conclusions}

Our results support the hypothesis of an independent correlation between NCN of BCR-ABL transcript at diagnosis and MMR at 18 months, sustained at 24 months. This observation has to be reinforced by a ROC analysis of a larger population. We showed that this correlation is independent with the Sokal score. This can be of major importance since Sokal, or Hasford scores, are currently the only prognostic factors at diagnosis [8] though they were established before the tyrosine kinase inhibitors era. At present, several therapeutic options are available to treat CML patients and the goal is to identify as soon as possible patients who might resist to imatinib and could benefit of alternative therapeutic strategies.

Our data suggest that a high NCN at diagnosis should be considered as a warning and may identify patients who could benefit of a straighter follow-up. If our results are confirmed on a larger series, they could be part of a new composite prognostic score available at diagnosis and in combination with the use of international conversion factors improve current scoring systems.

\section{REFERENCES}

[1] B. J. Druker, F. Guilhot, S. G. O'Brien, I. Gathmann, H. Kantarjian, N. Gattermann, M. W. Deininger, R. T. Silver, J. M. Goldman, R. M. Stone, F. Cervantes, A. Hochhaus, B. L. Powell, J. L. Gabrilove, P. Rousselot, J. Reiffers, J. J. Cornelissen, T. Hughes, H. Agis, T. Fischer, G. Verhoef, J. Shepherd, G. Saglio, A. Gratwohl, J. L. Nielsen, J. P. Radich, B. Simonsson, K. Taylor, M. Baccarani, C. So, 
L. Letvak and R. A. Larson, "Five-Year Follow-Up of Patients Receiving Imatinib for Chronic Myeloid Leukemia,” New England Journal of Medicine, Vol. 355, No. 23, 2006, pp. 2408-2417. doi:10.1056/NEJMoa062867

[2] H. Kantarjian, C. Sawyers, A. Hochhaus, F. Guilhot, C. Schiffer, C. Gambacorti-Passerini, D. Niederwieser, D. Resta, R. Capdeville, U. Zoellner, M. Talpaz, B. Druker, J. Goldman, S. G. O'Brien, N. Russell, T. Fischer, O. Ottmann, P. Cony-Makhoul, T. Facon, R. Stone, C. Miller, M. Tallman, R. Brown, M. Schuster, T. Loughran, A. Gratwohl, F. Mandelli, G. Saglio, M. Lazzarino, D. Russo, M. Baccarani and E. Morra, "Hematologic and Cytogenetic Responses to Imatinib Mesylate in Chronic Myelogenous Leukemia," New England Journal of Medicine, Vol. 346, No. 24, 2002, pp. 645-652. doi:10.1056/NEJMoa011573

[3] T. P. Hughes, J. Kaeda, S. Branford, Z. Rudzki, A. Hochhaus, M. L. Hensley, I. Gathmann, A. E. Bolton, I. C. van Hoomissen, J. M. Goldman and J. P. Radich, "Frequency of Major Molecular Responses to Imatinib or Interferon Alfa plus Cytarabine in Newly Diagnosed Chronic Myeloid Leukemia," New England Journal of Medicine, Vol. 349, No. 15, 2003, pp. 1423-1432. doi:10.1056/NEJMoa030513

[4] M. Baccarani, G. Saglio, J. Goldman, A. Hochhaus, B. Simonsson, F. Appelbaum, J. Apperley, F. Cervantes, J. Cortes, M. Deininger, A. Gratwohl, F. Guilhot, M. Horowitz, T. Hughes, H. Kantarjian, R. Larson, D. Niederwieser, R. Silver and R. Hehlmann, "Evolving Concepts in the Management of Chronic Myeloid Leukemia: Recommendations from an Expert Panel on Behalf of the European Leukemia Net,” Blood, Vol. 108, No. 6, 2006, pp. 1809-1820. doi:10.1182/blood-2006-02-005686

[5] M. Baccarani, J. Cortes, F. Pane, D. Niederwieser, G. Saglio, J. Apperley, F. Cervantes, M. Deininger, A. Grat- wohl, F. Guilhot, A. Hochhaus, M. Horowitz, T. Hughes, H. Kantarjian, R. Larson, J. Radich, B. Simonsson, R. T. Silver, J. Goldman and R. Hehlmann, "Chronic Myeloid Leukemia: An Update of Concepts and Management Recommendations of European Leukemia Net," Journal of Clinical Oncology, Vol. 27, 2009, pp. 6041-6051. doi:10.1200/JCO.2009.25.0779

[6] T. Hughes, M. Deininger, A. Hochhaus, S. Branford, J. Radich, J. Kaeda, M. Baccarani, J. Cortes, N. C. Cross, B. J. Druker, J. Gabert, D. Grimwade, R. Hehlmann, S. Kamel-Reid, J. H. Lipton, J. Longtine, G. Martinelli, G. Saglio, S. Soverini, W. Stock and J. M. Goldman, "Monitoring CML Patients Responding to Treatment with Tyrosine Kinase Inhibitors: Review and Recommendations for Harmonizing Current Methodology for Detecting BCR-ABL Transcripts and Kinase Domain Mutations and for Expressing Results,” Blood, Vol. 108, No. 1, 2006, pp. 28-37. doi:10.1182/blood-2006-01-0092

[7] D. Marin, D. Milojkovic, E. Olavarria, J. S. Khorashad, H. de Lavallade, A. G. Reid, L. Foroni, K. Rezvani, M. Bua, F. Dazzi, J. Pavlu, M. Klammer, J. S. Kaeda, J. M. Goldman and J. F. Apperley, "European Leukemia Net Criteria for Failure or Suboptimal Response Reliably Identify Patients with CML in Early Chronic Phase Treated with Imatinib Whose Eventual Outcome is Poor," Blood, Vol. 112, 2008, pp. 4437-4444.

doi:10.1182/blood-2008-06-162388

[8] M. Baccarani, G. Rosti, A. de Vivo, F. Bonifazi, D. Russo, G. Martinelli, N. Testoni, M. Amabile, M. Fiacchini, E. Montefusco, G. Saglio and S. Tura, "A Randomized Study of Interferon-Alpha Versus Interferon-Alpha and LowDose Arabinosyl Cytosine in Chronic Myeloid Leukemia,” Blood, Vol. 99, No. 5, 2002, pp. 1527-1535. doi:10.1182/blood.V99.5.1527 